\title{
Do elliptical galaxies have thick disks?
}

\author{
R C Thomson \\ Institute of Astronomy, Madingley Road, \\ Cambridge CB3 0HA, UK \\ A E Wright \\ Australia Telescope National Facility *, \\ PO Box 276, Parkes, N.S.W. 2870, Australia
}

February 18, 1990

\begin{abstract}
We discuss new evidence which supports the existence of thick disks in elliptical/S0 galaxies. Numerical simulations of weak interactions with thick disk systems produce shell structures very similar in appearance to those observed in many shell galaxies. We think this model presents a more plausible explanation for the formation of shell structures in elliptical/S0 galaxies than does the merger model and, if correct, supports the existence of thick disks in elliptical/S0 galaxies.
\end{abstract}

\section{Introduction}

The discovery of shell structures in elliptical galaxies (Malin and Carter 1980) was unexpected, and showed that these systems were not completely featureless. Malin and Carter (1983) estimated that about $17 \%$ of all elliptical galaxies had shell systems. More recently, Seitzer and Schweizer (1989) found that over half of the objects in a sample of 74 ellipticals/S0s exhibited shells. Shell structures are therefore not rare they are a common phenomena in elliptical galaxies.

The currently accepted model for shell formation is by merger with another galaxy (Quinn 1984, Dupraz and Combes 1986, Hernquist and Quinn 1988). Several problems are noted by Thomson and Wright (1990). Briefly, in order to explain asymmetric shell structures, the merger model requires a head-on collision along the major axis of the primary galaxy, viewed nearly perpendicular to the line of impact, with another galaxy which has a similar stellar population to the that of the primary galaxy and which somehow produces shell structures with a radial brightness distribution similar

"The Australia Telescope National Facility is operated in association with the Division of Radiophysics by CSIRO. 
to that of the underlying galaxy. We think the combination of all these factors makes this explanation of what is now known to be a relatively common phenomenon, highly improbable.

\section{A weak interaction model for shell galaxies}

Numerical simulations of interacting thick disk systems (Thomson and Wright 1990) have shown that shell structures can be readily formed by this process. In these simulations, the thick disk is represented by a quasi-spherical distribution of massless test particles, with both the primary and secondary galaxies modelled by spherical Plummer potentials, and the interaction characterized by a parabolic orbit of the secondary. The test particles are set in motion at a tangent to their radius vector with a speed equal to their appropriate circular velocity, and the position of each particle is calculated under the influence of the combined time varying potential.

When such an interacting system is viewed along the effective axis of rotation, the induced stellar density wave is seen to have a spiral morphology (Fig.1; top row). When such a system is viewed nearly edge on, asymmetric shell structures are clearly visible (Fig.1; middle row). The asymmetry is more pronounced when the orbit of the secondary galaxy cuts through the simulated thick disk.

In the classification scheme described by Prieur (1989), type 1 shell systems are interleaved and well aligned along the major axis of the elliptical. According to our model, type 1 shell systems arise in elliptical galaxies which have a thick disk and a conspicuous spiral density wave viewed approximately edge on. When such a thick disk system is viewed more face-on, the density wave is seen to form fuller, but not complete arcs, and the shells also exhibit some spirality (Fig.1; bottom row). We associate this projection with type 2 ('all-round') shell systems. Furthermore, the observations that elongated shell galaxies are preferentially surrounded by type 1 shell systems, and that round-like shell galaxies are preferentially surrounded by type 2 systems (Prieur 1989) imply that these ellipticals are oblate spheroids when interpreted in terms of our model.

Shell galaxies that cannot be classified as type 1 or 2 , are classified as type 3 , which includes those galaxies with too few shells or which have complex structure (eg, Fornax A). In our model, galaxies with only a small number of shells may occur soon after the encounter (before the density wave has wound up by differential rotation). At the present stage of our modelling, we are unable to reproduce complex shell structures, probably because of the oversimplified assumptions of our model. However, we think that these complex shell systems are also caused by (possibly strong) interactions since these are observed in galaxies which are otherwise known to be interacting (eg, NGC1549/1553, NGC1316(Fornax A)/NGC1317). 


\section{Thick disks in elliptical/S0 galaxies}

Featureless thick disks (ie, with no conspicuous spiral density wave) could exist in all elliptical galaxies without being observed. As such, most of the angular momentum in these systems could remain hidden. It is interesting to note that most of the elliptical galaxies in Malin and Carter's catalog of shell galaxies (Malin and Carter 1983) have been classified as S0 by Thronson et al. 1989. If this reclassification is correct, considerable angular momentum must be present in these (possibly thick disk) systems.

Detailed surface photometry of elliptical galaxies has revealed significant deviations from perfect ellipses (Jedrzedjewsi 1987, Franx 1988). In this respect, pointed isophotes (positive $\cos 4 \theta$ term) are thought to indicate the presence of a thin disk. We think that 'boxy' isophotes (negative $\cos 4 \theta$ term) may be the signature of thick disks in oblate spheroids viewed edge on. The extremely box-shaped elliptical IC3370 has been observed by Jarvis (1987). The maximum rotation velocity on the major axis is in good agreement with theoretical models of an oblate spheroid supported by rotation alone. For this reason, Jarvis proposed classifying this galaxy as S0pec. We think this galaxy is not peculiar, but just an extreme case of an elliptical galaxy with a featureless, but easily detectable, thick disk.

\section{Discussion}

The existence of thick disks in elliptical galaxies is postulated on the evidence of regular shell structures seen in a large number of these systems. By analogy with thin disk systems, if the thick disks extends much further than the visible bulge, elliptical galaxies may have much more angular momentum than previously thought. The stellar orbits in the outer regions of these galaxies could then be dominated by rotation. (NB. The shell system in NGC3923 extends more than $80 \mathrm{kpc}$ from the galaxy centre!) Due to the extremely low surface brightness in these regions, only thick disk systems with identifiable features (shells) could have reliable velocities measured, and even then only with great difficulty.

It is interesting to note that our thick disk model can explain the observed connection between shell morphology (type 1 vs type 2) and roundness in terms of a simple projection effect. The merger model cannot explain this connection in a unified way - the differences must be modelled by different initial conditions, and cannot be explained by projection effects alone (Dupraz and Combes 1986).

All galaxies interact to some extent, and our model only requires the existence of a dynamically cool population of stars in nearly circular orbits to produce shells similar to those seen in many elliptical/S0 galaxies. Our simulations show that only weak interactions with galaxies $1 / 10$ th the mass of the primary and at distances $\sim 40 \mathrm{kpc}$ are needed to produce visible shell structures. Taking into account the small impact parameter and small relative velocity required by the merger model, the cross section 


\begin{tabular}{|c|c|c|c|c|c|}
\hline 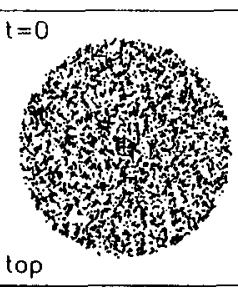 & 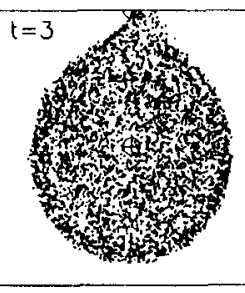 & 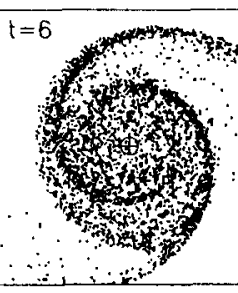 & 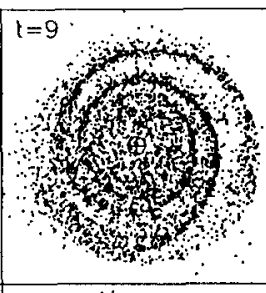 & 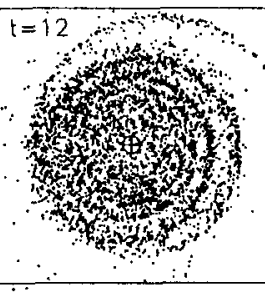 & 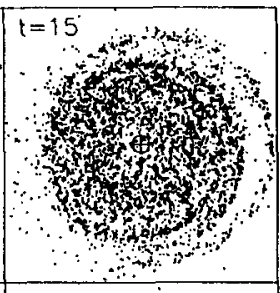 \\
\hline 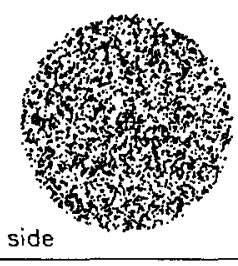 & 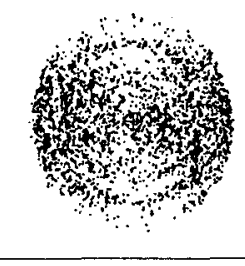 & 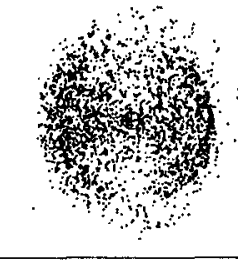 & 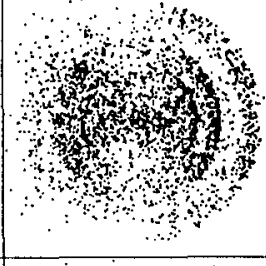 & W & $\frac{1}{4}$ \\
\hline 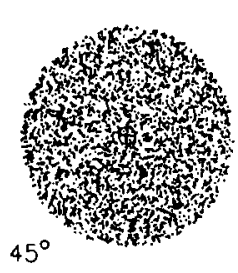 & 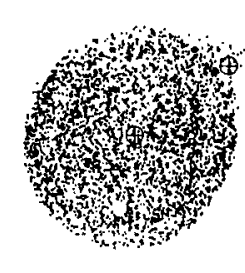 & 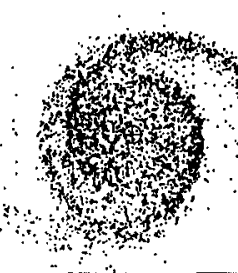 & 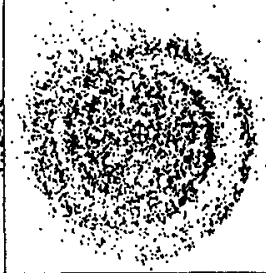 & $\begin{array}{l}3 \\
3 \\
3\end{array}$ & w \\
\hline
\end{tabular}

Figure 1: Weak interaction model simulation with 10000 particles and a secondary galaxy 1/10th mass of primary. Time unit $\sim 10^{9}$ years; initial radius of galaxy $80 \mathrm{kpc}$. Top row - view from above; middle row - view from front; bottom row - view from $45^{\circ}$. For clarity, only the band of particles from the projected equatorial region $\left(\left|z^{\prime}\right|<0.2\right)$ is shown.

for producing shell structures by weak interactions in thick disks is many orders of magnitude greater than the cross section for shell production by merger.

In conclusion, we think that at least some elliptical galaxies have thick disks and that shell systems are formed in these thick disks by weak interaction with another galaxy.

\section{$5 \quad$ References}

Dupraz,C., \& Combes,F., 1986. Astr. Astrophys, 166, 53.

Franx,M., 1988. Ph.D. Thesis, Leiden.

Hernquist,L., \& Quinn,P.J., 1988. Astrophys. J., 331, 682.

Jarvis,B.J., 1987. IA U Symp. No. 127, ed. De Zeeuw,T., Reidel, Dordrecht.

Jedrzejewski,R.I., 1987. IA U Symp. No. 127, ed. De Zeeuw,T., Reidel, Dordrecht.

Malin,D.F., \& Carter,D., 1980. Nature, 285, 643.

Malin,D.F., \& Carter,D., 1983. Astrophys. J., 274, 534.

Prieur,J.-L., 1989. European Southern Observatory Scientific Preprint.

Quinn,P.J., 1984. Astrophys. J., 279, 596.

Seitzer,P., \& Schweizer.F., 1989. Preprint.

Thomson,R.C., \& Wright,A.E., 1990. Cambridge University Astronomy Preprint.

Thronson,H.A., Bally,J. \& Hacking,P., 1989. Astron. J., 97, 363. 